\title{
EFFECT OF CORTISONE ON TISSUE CULTURES
}

\author{
BY
}

\section{A. STAFFORD STEEN}

\author{
Department of Pathology, Institute of Ophthalmology, London
}

NUMEROuS recent reports shọ that cortisone apparently exerts an inhibitory effect, both clinically and in experimental animals, on mesenchymal growth; the evidence on its effect upon epithelial cells is less conclusive. This work has been reviewed by Duke-Elder and Ashton (1951).

The object of the experiments here reported is to elucidate the action of cortisone on growth in tissue cultures and to ascertain whether any effect it may have is direct or indirect in its action. Little work on this aspect has been published. The effect of cortisone upon epidermal cells of the mouse has been studied by Green and Ghadially (1951); they found that cortisone was a powerful inhibitor of mitosis in the pre-prophase stage. Cornman (1951) states that desoxycorticosterone acetate (DOCA) and cortisone acetate selectively damage fibroblasts without in any way affecting epithelial growth. These results, however, were obtained by replacing the fluid phase of the cultures by a solution of DOCA in saline, an apparently nonnutrient medium. Furthermore, he found that if 10 per cent. of the saline were replaced by serum no such effect on the tissue cultures was seen up to concentrations of $0.03 \mathrm{mg}$. $/ \mathrm{ml}$. DOCA or up to $0.026 \mathrm{mg} . / \mathrm{ml}$. cortisone acetate. This suggests that unless the conditions are quite artificial, cortisone acetate does not, in fact, suppress the growth of the cells. Barber and Delaunay (1951) injected guinea-pigs with large doses of cortisone, and found that plasma from these animals inhibited the growth of fibroblasts in tissue culture. Their experimental results cannot be regarded as conclusive, however, for only eleven of twenty specimens of plasma showed this effect and the large doses of cortisone produced severe constitutional disturbances in the animals, some even dying before the blood could be taken. Holden and others (1951) studied the effect of cortisone on tissue cultures of spleens from mice, guinea-pigs, and rabbits. The highest concentrations of cortisone they used (which were only $1 / 100$ th of the lowest concentration used in our experiments) produced moderate or marked inhibition of fibroblastic growth, whereas their lower concentrations had no such effect. It is obvious that further work on this question is desirable. 


\section{EXPERIMENTS}

In testing the effect of cortisone on tissue cultures the experiments were divided into three groups.

Group 1.-Cortisone was added to the tissue cultures in concentrations comparable to that of the therapeutic level generally used in man. The tissue cultures in this group contained $0.002 \mathrm{mg} . / \mathrm{ml}$. to $0.05 \mathrm{mg} . / \mathrm{ml}$., i.e. from 2 to 25 times the usual therapeutic level.

Group 2.- In this group very high concentrations of cortisone were used, the tissue cultures containing from $0.5 \mathrm{mg} . / \mathrm{ml}$. to $2 \mathrm{mg}$. $/ \mathrm{ml}$., i.e. 500 to 2,000 times the therapeutic level.

Group 3.-Plasma or serum from fowls and patients injected parenterally with cortisone was added to the tissue culture.

The usual hanging-drop tissue culture method was used during most of these experiments. Heart muscle, lungs, and intestines from 11- to 14-day-old chick embryos were used. Tissue specimens $1 \mathrm{~mm}$. square were embedded in a mixture of embryonic extract from 11-day-old chick embryos and fowl plasma. Cortisone-acetate suspension (Merck), in varying concentrations, was added to this mixture. The original suspension, which contained $25 \mathrm{mg}$. $/ \mathrm{ml}$., was diluted by the addition of Gey's saline solution. In order to prevent separation of the cortisone, the suspension was mixed thoroughly immediately before it was added to the culture. In control experiments Gey's solution was used in place of cortisone suspension.

The drops of plasma, embryonic extract, and cortisone dilution were then thoroughly mixed on the coverslip and spread out to form a circular disk, into the middle of which a piece of tissue was placed. When coagulation had taken place, the coverslip was placed on a depression slide, sealed, and incubated at $38^{\circ} \mathrm{C}$.

In most cases the tissues were transplanted three times before being used for experiments. The final transplant was grown for 72 hours and then divided into four equal parts - two for use in the experiment and two for use as controls. Each experiment was performed with 12 to 36 cultures, and altogether several hundred hanging-drop cultures were examined.

The following media were used in these experiments:

(1) Fowl-plasma to which cortisone-acetate suspension was added in varying concentrations. The controls were set up with the same fowl-plasma, and the cortisone suspension was replaced by Gey's saline solution.

(2) Fowl-plasma from a 1-year-old hen injected intra-muscularly with cortisone. In the first case the hen received $0.25 \mathrm{ml}$. cortisone acetate (Merck) over 24 hours, and 3 hours after the last injection it was bled from the wing-vein. In the later series the same hen received $1 \mathrm{ml}$. ( $25 \mathrm{mg}$.) cortisone acetate 4-hourly and was bled 3 hours after the last injection.

When bleeding the birds, an addition was made of heparin ( 5 international units per ml. blood), which facilitated the handling of the blood and had no detrimental effect on the tissue growth.

(3) Serum from patients under prolonged treatment with cortisone for rheumatoid arthritis. For controls serum from a healthy human was taken. In each case the serum was obtained by centrifugalization of the unadulterated clotted blood. 


\section{RESULTS}

In the experiments of Group 1, wherein moderate concentrations of cortisone were used ( 2 to 25 times the normal therapeutic level), no appreciable difference was observed between the growth of the tissue cultures containing cortisone and that of the controls. All these cultures showed approximately equal numbers of mitotic figures after 24 to 48 hours' growth and reached the maximum size usually seen in hanging-drop cultures after 72 hours.

In one experiment there appeared to be marked delay of growth in the cortisone series in the first 24 hours, but during the following 24 hours' incubation, growth increased and appeared to reach the maximum size after 72 hours. This delay was not observed in later experiments and may have been caused by some coincidental factor.

In the experiments of Group 2, wherein very large concentrations of cortisone were used (500 to 2,000 times that of the usual therapeutic level), there was some inhibition of growth which was sometimes complete. In the highest concentration, when growth did occur the cells appeared prematurely aged; they showed vacuoles in the cytoplasm and growth stopped after a period of 12 to 48 hours. Nevertheless, a reasonably good growth was seen up to concentrations of $0.5 \mathrm{mg} . / \mathrm{ml}$., and occasionally even at 1 and $2 \mathrm{mg} . / \mathrm{ml}$., as shown in Figs 1 and 2. Mitotic figures were visible and the majority of the cells showed numerous vacuoles in the cytoplasm, an unusual feature after only 48 hours' incubation. It is obvious that there is no question of an arrest of mitosis, as occurs, for example, in the presence of colchicine, and the fact that mitosis is carried through to maturation is shown by the absence of mitotic figures in the fully grown 72-hours' cultures. Figs 3 and 4 show vigorous mitosis after $36-48$ hours incubation in fibroblastic growth containing the high concentration of $0.5 \mathrm{mg} . / \mathrm{ml}$. cortisone. Several stages of mitosis can be seen. After 72 hours, the growth of the mass of fibroblasts in the series with lower cortisone concentrations reached maximum size; when, however, the higher concentrations were used, the extent of growth was smaller and often rudimentary. Cultures of epithelial tissue showed a similar reaction. A 48-hour tissue culture of a piece of small intestine from a 14-day-old chick embryo embedded in medium containing the very large amount of $1.5 \mathrm{mg} . / \mathrm{ml}$. showed abundant epithelial growth (Figs 5 and 6).

In the experiments of Group 3, in which the plasma of a fowl which had been injected with cortisone, and the serum of patients undergoing intensive cortisone treatment were used; no inhibitory action on the growth of the tissue cultures was demonstrated.

An interesting phenomenon, which has already been reported elsewhere (Steen, 1951), was observed during the course of these experiments. When the stock suspension of cortisone acetate is added to the plasma-drop, a turbidity develops and crystals of the 


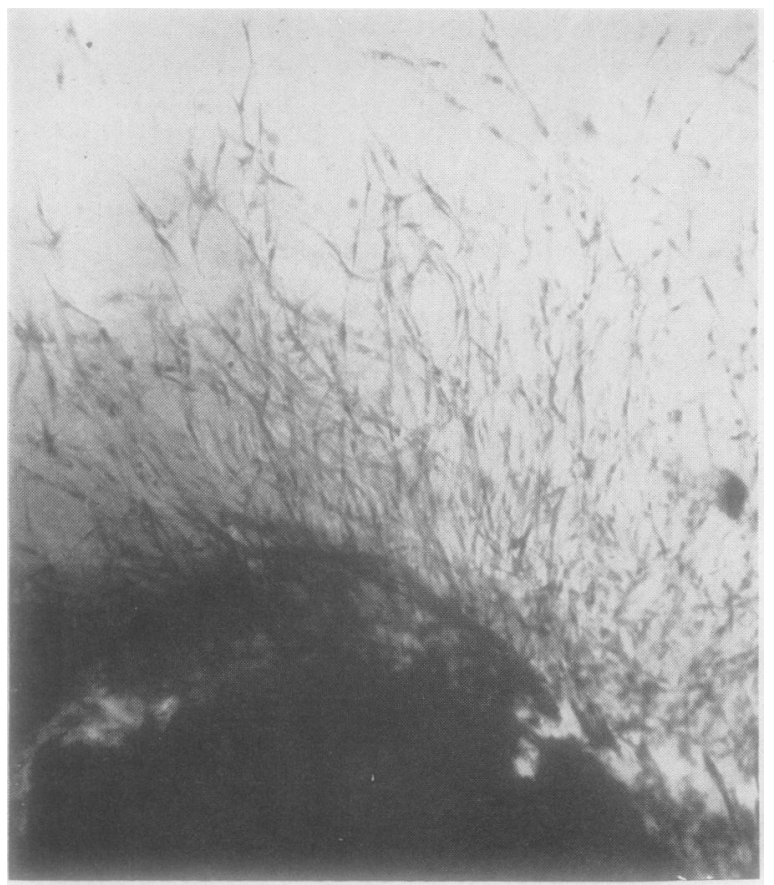

FIG. 1.-Fibroblastic newgrowth from chick embryo heart after 48 hours' incubation. Cortisone concentration of the medium 0.5 mg. $/ \mathrm{ml} . \quad \times 56$.

FIG. 2.-Fibroblastic newgrowth as in Fig. 1, but cortisone concentration $2 \mathrm{mg}$. $/ \mathrm{ml}$. $(2,000$ times therapeutic level). 56.

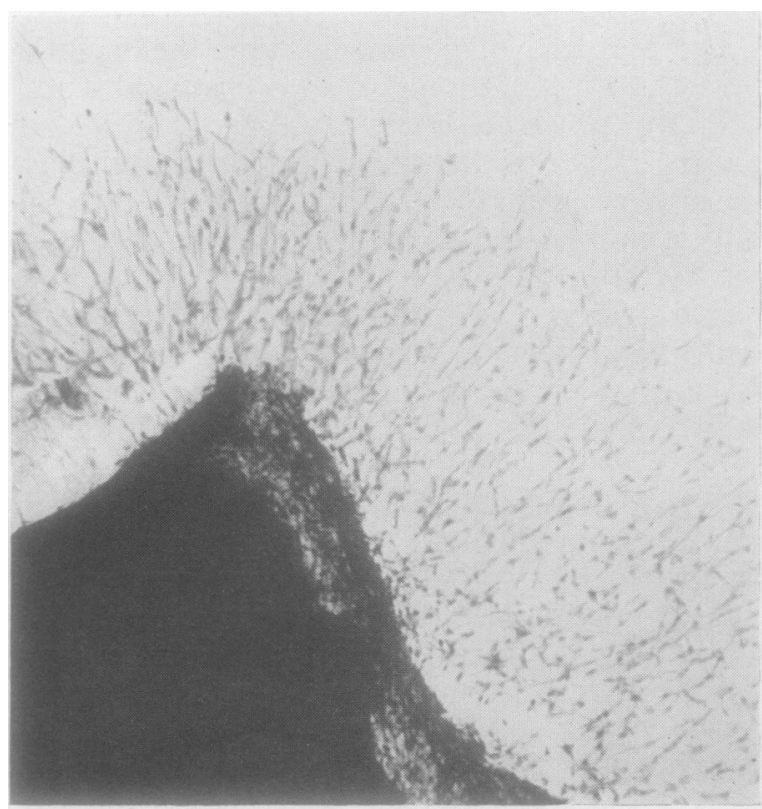




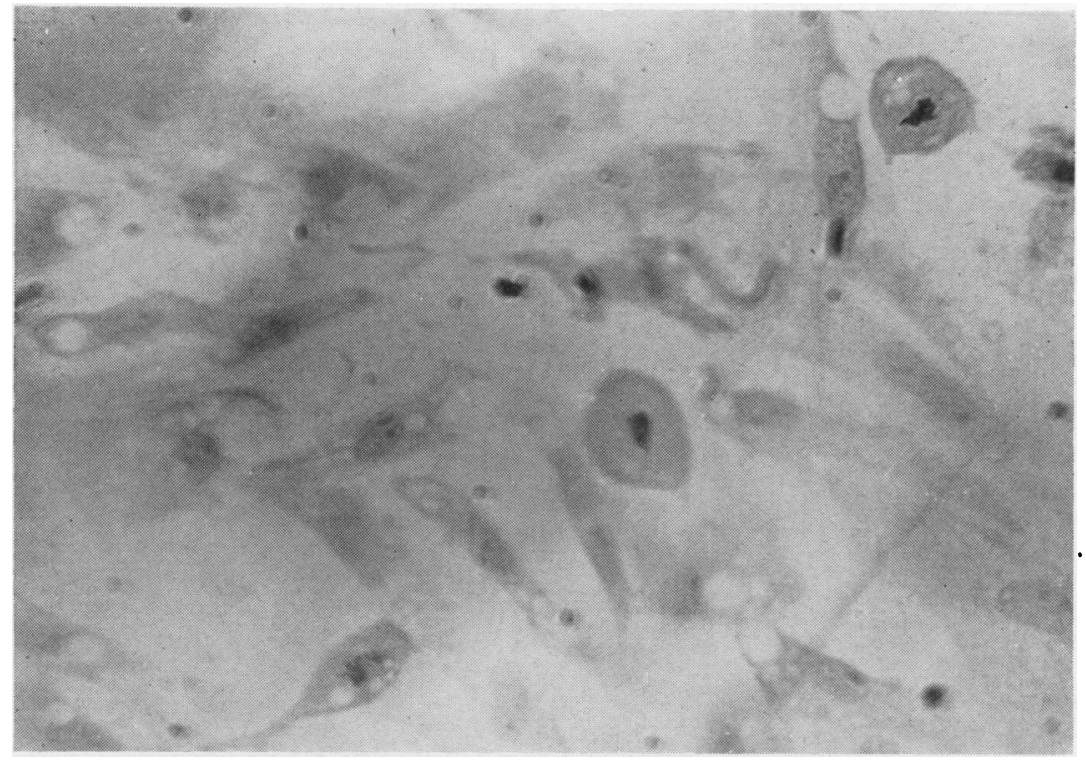

Fig. 3.-Fibroblasts grown in a medium containing $0.5 \mathrm{mg}$./ $\mathrm{ml}$. cortisone acetate. In a single field three cells may be seen undergoing mitosis.

$\times 530$.

Fig. 4.-Fibroblasts grown in a medium containing $0.5 \mathrm{mg}$. $\mathrm{ml}$. cortisone acetate. After 48 hours' incubation the cells are undergoing mitotic division.

Numerous vacuoles may be seen in the cytoplasm. $\times 1,000$. 


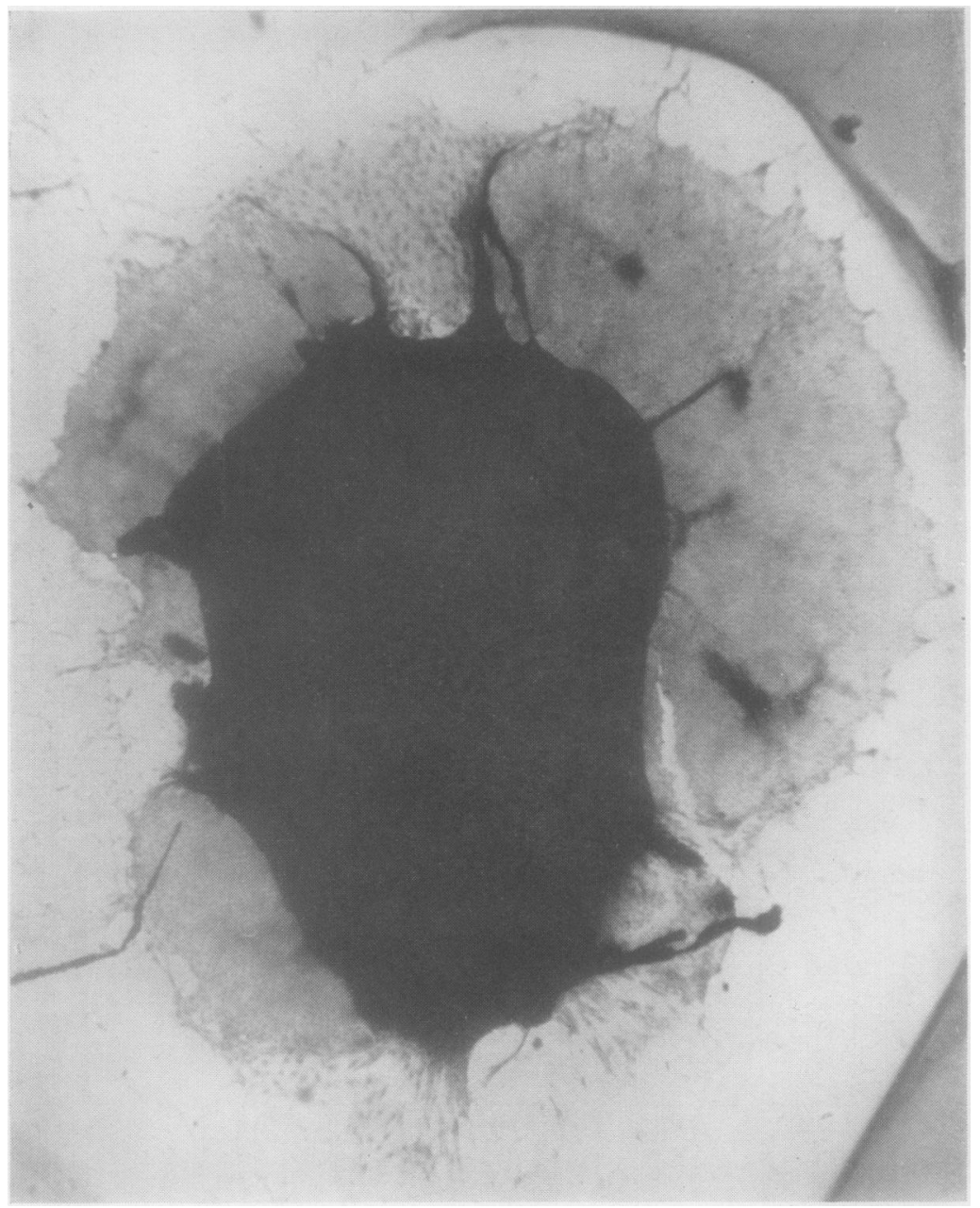

FIG. 5.-Tissue culture from small intestine of chick embryo grown in medium containing $1.5 \mathrm{mg} . / \mathrm{ml}$. cortisone, showing large wing-shaped out-growth of epithelium. At the upper and lower pole sparse fibroblastic growth may be seen. 48 .

cortisone acetate may be seen, under the microscope, discretely suspended in the otherwise clear plasma. If a tissue culture is now implanted into this suspension and incubated, the crystals nearest to the tissue dissolve after some 5 hours and a clear, crystal-free zone gradually develops around the implant (Fig. 7). It is important that this phenomenon was found to depend on the presence of glucose; if this carbohydrate is exhausted, these changes are arrested, and, if glucose is progressively added, the extent of the zone of clearing correspondingly increases (Fig. 8). 


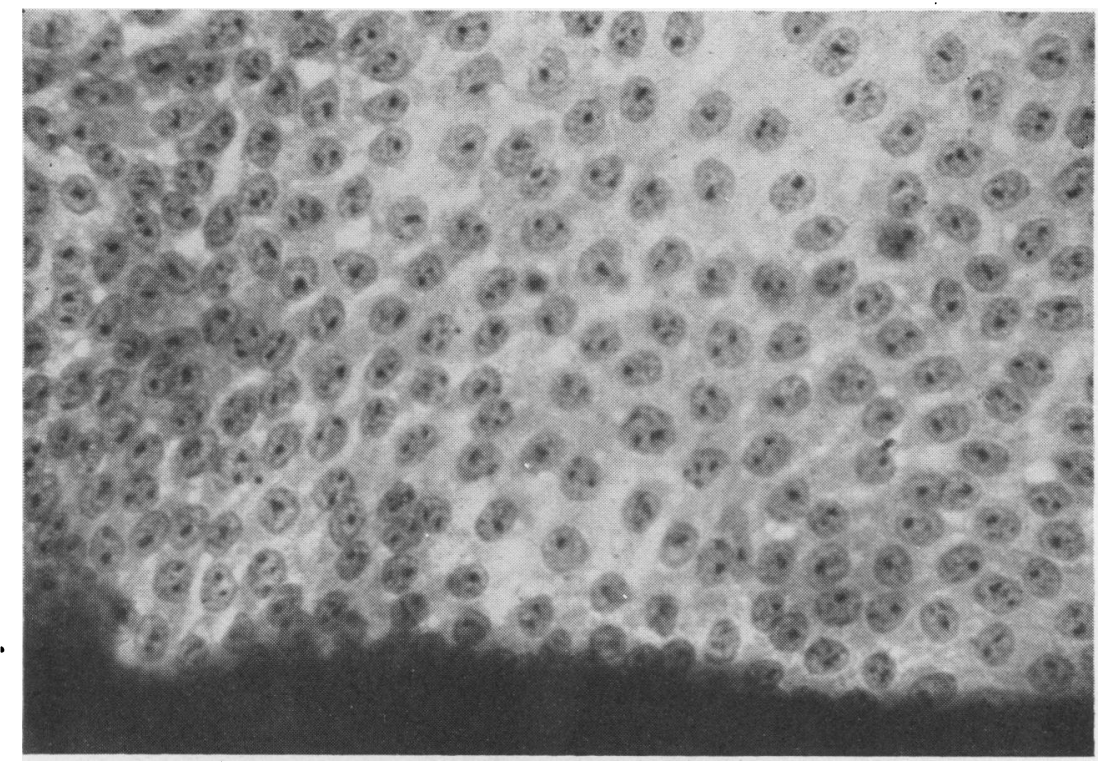

FIG. 6. - High-power view of epithelial newgrowth shown in Fig. 5. $\quad \times 600$.

\section{Discussion}

The experiments described above show that, when tissue culture is carried out in the presence of cortisone in concentrations comparable to and even 25 times greater than the usual therapeutic levels, no suppression of growth nor inhibition of mitotic activity is demonstrable, either in the fibroblasts or in the epithelial cells grown from chick embryos. Only when the tissue cultures were exposed to larger amounts of cortisone far in excess of therapeutic concentration, did signs of early exhaustion become evident, so that growth was scanty and sometimes completely inhibited. Such an inhibition, however, can receive other explanations than the action of cortisone per se. It must be agreed that the conditions of the experiments wherein the high concentrations of this hormone were used, were grossly abnormal in that the ionic balance of the culture medium must have been greatly disturbed, while the reaction between the cortisone acetate and the glucose would presumably result in a starvation of the growing cells. Nevertheless, the facts that several of the cultures, both fibroblastic and epithelial, showed some growth in spite of these adverse factors, and that all cultures exposed to a lesser concentration of cortisone were unaffected, can only mean that cortisone has little if any direct action on fibroblastic or epithelial cells in tissue culture.

It is difficult to apply these results directly to tissue growth in the 
intact animal, but the demonstration that the plasma of the fowl injected with cortisone, and the serum of the patients under cortisone treatment, failed to inhibit tissue-growth, suggests that no intermediate inhibitor, which in turn acts upon the tissue culture, is

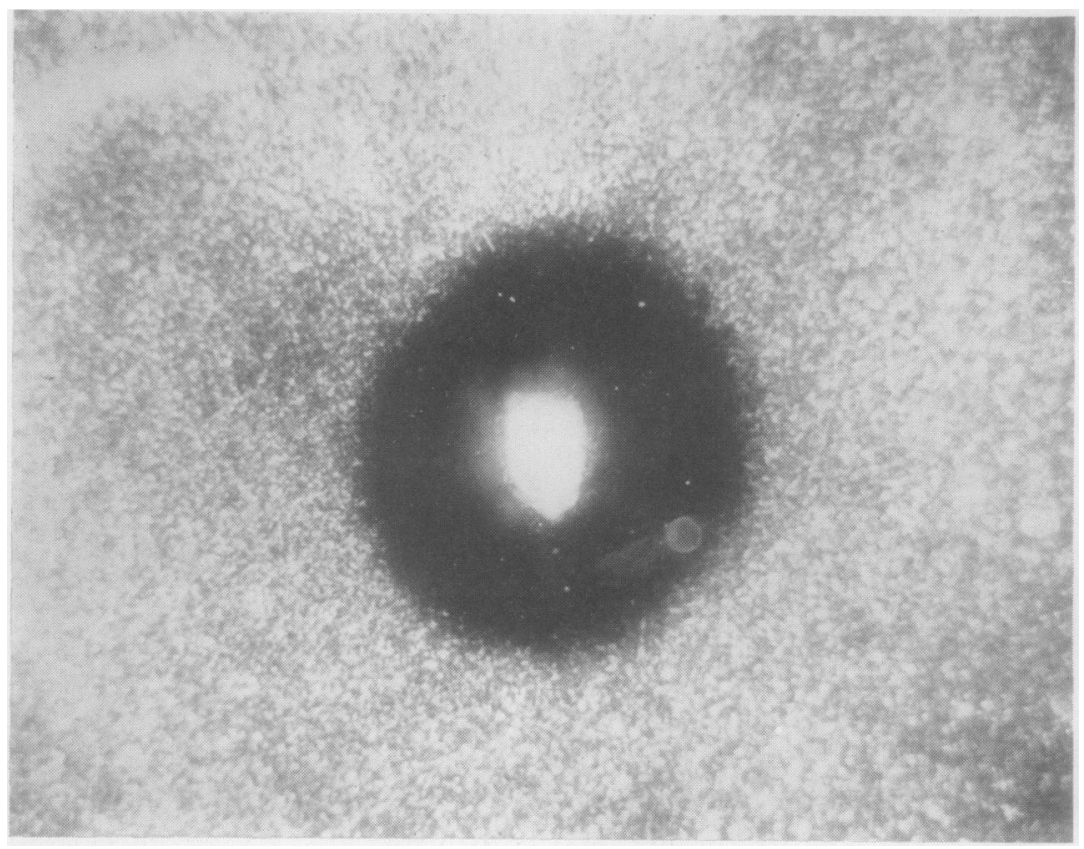

FIG. 7.--Tissue culture growing in cortisone suspension. After 5 hours the crystals nearest to the tissue dissolved and a clear zone developed around the growth. Photographed in reflected light. $\times 15$.

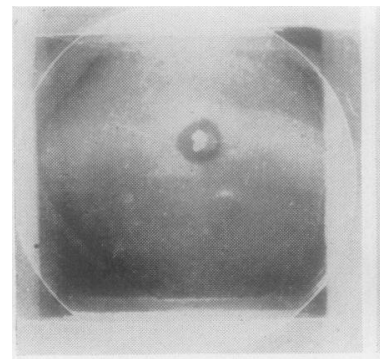

(a)

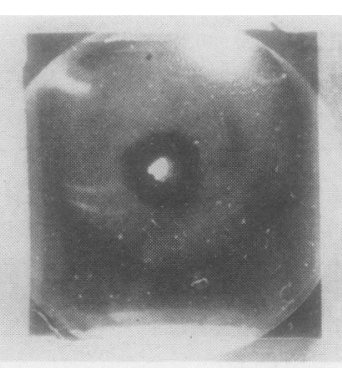

(b)

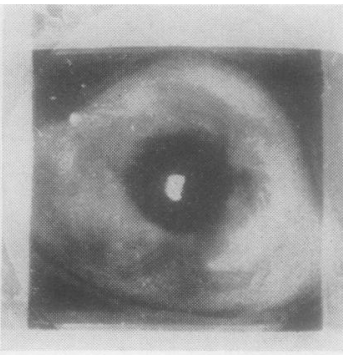

(c)

FIG. 8.--Three tissue cultures growing in cortisone suspension showing the effect of varying concentrations of glucose. (a) contains the normal glucose addition in the media, $(b)$ twice this amount, $(c)$ four times this amount. The extent of the clearing area is seen to bear a direct relationship to the quantity of glucose in the medium. 
elaborated or released into the blood stream. It is probable, therefore, that the undoubted inhibitory action of cortisone upon fibroblastic activity, which has been amply confirmed both clinically and experimentally (Duke-Elder and Ashton, 1951), must be explained by some indirect mechanism acting at the cellular level in the living organism. It is possible that the insoluble cortisone acetate becomes available for this purpose through the formation of a soluble compound formed by combination with glucose.

\section{SUMMARY}

Tissue culture experiments were carried out on fibroblastic and epithelial growth from chick embryos to test the effect of cortisone acetate.

It was found that cortisone had no inhibitory effect upon tissue culture in concentrations comparable to and even 25 times greater than the usual therapeutic levels. There appeared to be no disturbance in the normal process of mitosis.

Growth was, however, inhibited by doses far in excess of therapeutic concentration, but this effect may be explained by the operation of other factors.

In the course of the experiments, it was found that, in the presence of living cells, cortisone-acetate crystals undergo a chemical change by combining with glucose to form a new and soluble compound which may be cortisone-glucoside.

I wish to thank Sir Stewart Duke-Elder and Dr. Norman Ashton for their invaluable assistance in the preparation of this paper. My thanks are also due to Dr. F. Dudley Hart for the provision of serum from patients receiving cortisone treatment, to Dr. Peter Hansell of the Medical Illustration Department for photographs, and to Mr. D. Bedford for technical assistance.

The cortisone used in these experiments was supplied by the Medical Research Council and was in part derived from a gift from Merck and Co. made jointly to the Medical Research Council and the Nuffield Trust.

\section{REFERENCES}

Barber, M., and Delaunay, A. (1951). J. Path. Bact., 63, 549.

CORNMAN, I. (1951). Science, 113, 37.

Duke-Elder, S., and AsHTon, N. (1951). British Journal of Ophthalmology, 35, 695.

Green, H. N., and Ghadially, F. N. (1951). Brit. med. J., 1, 496.

Holden, M., Seegal, B., and Ryby, I. (1951). Amer. J. Path., 27, 748.

SteEn, A. S. (1951). Nature, Lond., 168, 152. 